\title{
The Epistemic Value of Expert Autonomy
}

\author{
Finnur Dellsén \\ Forthcoming in Philosophy and Phenomenological Research
}

\begin{abstract}
According to an influential Enlightenment ideal, one shouldn't rely epistemically on other people's say-so, at least not if one is in a position to evaluate the relevant evidence for oneself. However, in much recent work in social epistemology, we are urged to dispense with this ideal, which is seen as stemming from a misguided focus on isolated individuals to the exclusion of groups and communities. In this paper, I argue that that an emphasis on the social nature of inquiry should not lead us to entirely abandon the Enlightenment ideal of epistemically autonomous agents. Specifically, I suggest that it is an appropriate ideal for those who serve as experts in a given epistemic community, and develop a notion of expert acceptance to make sense of this. I go on to show that, all other things being equal, this kind of epistemic autonomy among experts makes their joint testimony more reliable, which in turn brings epistemic benefits both to laypeople and to experts in other fields.
\end{abstract}

\section{INTRODUCTION}

You and I both believe that the Earth was formed billions of years ago. And yet neither of us has directly examined any of the scientific evidence for that claim, or indeed for the various theories and models that underwrite it. In this case, as in so many other cases, our opinions are based on the testimony of experts. In an influential article, Goldman (2001) considered the issue of how laypeople should form and modify their opinions in response to the 
testimony of such experts. Accordingly, much of the literature has focused on epistemological problems about expert testimony from the point of view of laypeople, i.e. non-experts. ${ }^{1}$ However, the same issue can also be considered from the point of view of the experts themselves. In this paper, we will be concerned with how (if at all) an expert's own opinions should be influenced by the opinions of other experts within the same area of specialization. Should these experts treat each other in much the same way as laypeople should treat experts - i.e. as sources of information on the basis of which to form new opinions and modify existing ones?

In considering this question, one is immediately struck by two prima facie plausible but seemingly conflicting considerations. On the one hand, there is a common presumption that inquiring minds should not rely on other people's say-so, at least not if they are in a position to evaluate the relevant evidence for themselves. The importance of this kind of intellectual independence has been emphasized by several influential Enlightenment thinkers including Descartes, Locke, and Kant. ${ }^{2}$ Indeed, this common sentiment appears especially strong when it is directed at experts, such as scientists and other scholars, who are frequently accused of 'groupthink' and 'herd mentality'. Accusations of this sort assume that experts should strive to be epistemically autonomous in something like the following sense:

Epistemic autonomy: $S$ is epistemically autonomous with regard to a proposition $P$ to the extent that $S$ 's epistemic attitude to $P$ is not directly

\footnotetext{
${ }^{1}$ In Goldman's terminology, the literature has concerned the "novice/expert problem" as opposed to "expert/expert problem" (Goldman, 2001, esp. 89-90). For recent discussion of the former, see e.g. Coady (2006), Collins and Evans (2007), Lane (2014), and Martini (2014).

${ }^{2}$ Rule III in Descartes' Rules for the Direction of the Mind $(1628,13)$ explicitly forbids inquiring minds from relying on "what other people have thought". Similarly, Locke (1689, I, iv, 23) claims that "[t]he floating of other men's opinions in our brains, makes us not one jot the more knowing, though they happen to be true." Finally, Kant $(1784,54)$ goes so far as to identify the Enlightenment with the tendency to think for oneself:
}

Enlightenment is man's emergence from his self-incurred immaturity. Immaturity is the inability to use one's own understanding without the guidance of another. [...] The motto of enlightenment is therefore: Sapere aude! Have courage to use your own understanding! 
influenced by other agents' epistemic attitudes to $P{ }^{3}$

On the other hand, it seems obvious that if laypeople should treat expert opinion as a source of information, then so should other experts. For example, if an expert $X_{1}$ has not yet made up her mind about some new hypothesis $H$ within her domain of specialization $D$, and then learns that her generally-trustworthy colleague $X_{2}$, who also specializes in $D$, strongly endorses $H$, it would seem that $X_{1}$ should take that as a reason to endorse $H$ as well. ${ }^{4}$ However, this apparently reasonable procedure seems to conflict with epistemic autonomy since it clearly involves $X_{1}$ 's opinion about $H$ being directly influenced by another person's opinion about $H$, viz. $X_{2}$ 's.

One of the things I hope to do in this paper is to suggest a more sophisticated conception of epistemic autonomy that eliminates the conflict between these two considerations. In particular, I will appeal to a version of the wellknown distinction between belief and acceptance (Cohen, 1992) to argue that the ideal of epistemic autonomy should not be seen as applying to belief, but instead to a kind of expert acceptance (section 2). Having clarified the relevant notion of epistemic autonomy in this way, I spend the rest of the paper exploring what sort of rational basis, or rationale, there could be for the ideal of epistemic autonomy among experts (section 3). Roughly, my suggestion will be that epistemic autonomy is a kind of social good - something that is valuable for its effects on communities of truth-seeking agents - rather than something that individual agents benefit from directly. I argue for this by showing that, all other things being equal, agreement in a group of epistemically autonomous experts is a more reliable guide to truth than an otherwise identical agreement in a group of non-autonomous experts.

Although my approach in what follows will abstract away from any par-

\footnotetext{
${ }^{3}$ This is a preliminary definition of epistemic autonomy that captures the spirit of what I will be concerned with in this paper. In section 2, I clarify and modify the definition in several ways; in section 4, I furthermore provide a probabilistic analysis of epistemic autonomy.

${ }^{4}$ Indeed, epistemological theories of testimony on both sides of the reductionism/antireductionism divide (see, e.g., Coady, 1992; Fricker, 1994) would seem to imply that in such a situation, $X_{1}$ 's opinion should be at least somewhat influenced by $X_{2}$ 's opinion - whether that's because $X_{1}$ has independent reasons to trust $X_{2}$ (as per reductionism) or because $X_{1}$ is a priori entitled to trust $X_{2}$ (as per anti-reductionism).
} 
ticular kinds or domains of expertise, I will take the scientific expert as a paradigm case of the kind of expert with which I am concerned. There are a few different reasons for this. First, the kind of expert that interests me is one that non-experts can identify relatively easily; and I'll assume that scientific experts can be identified by appealing to their academic qualifications, research output, or other relatively transparent indicators. Second, the experts I am concerned with will be able to provide testimony to non-experts about the truth-value of theories or hypotheses; and I take it that scientific experts - perhaps in contrast to, e.g., expert craftspeople - are generally able to provide such testimony. Third, my kind of experts will have a limited and relatively well-defined domain of expertise, such that they too will need to rely on other experts when reaching beyond that domain; again, I take this to be true of scientific experts (at least in contemporary science). That said, what I have to say below about the value of epistemic autonomy among scientific experts applies equally to other kinds of experts in so far as they satisfy these conditions.

\section{The Ideal of Expert Autonomy}

Before we start exploring why it would be valuable for experts to be epistemically autonomous, we must first say more about what it would be for experts to be autonomous in the epistemic sense. I will approach this task by considering two potential objections to the idea that experts should be epistemically autonomous, and then show how the specific conception of epistemic autonomy that I have in mind avoids these objections. Most of the section concerns the objection that being epistemically autonomous cannot be epistemically rational, since it involves deliberately ignoring other people's beliefs even when they are clearly relevant to the truth-value of one's own beliefs. I begin, however, with a more fundamental challenge, viz. that it would often be impossible for experts to remain uninfluenced by their fellow experts. In many disciplines, experts typically work together in groups and are constantly

\footnotetext{
${ }^{5}$ To be sure, the extent to which this is true varies widely from one discipline to the next. For example, collaboration is less common in mathematics and purely theoretical physics, but even there the trend seems to be towards increasingly collaborative endeavors.
} 
communicating with each other. ${ }^{5}$ So how could epistemic autonomy among experts be anything other than a pipe-dream, inapplicable in most, if not all, real circumstances?

In response, let me clarify the notion of 'epistemic autonomy' in three important ways. First, note that our preliminary definition of epistemic autonomy holds that an agent is epistemically autonomous with regard to a proposition $P$ to the extent that her epistemic attitude to $P$ is not directly influenced by other agents' epistemic attitudes to $P$. This is meant to exclude the indirect kind of influence someone can have over another's opinions in virtue of presenting that person with evidence or arguments for $P$ that she evaluates herself. This latter kind of influence would be indirect in that the agent in question would make up her own mind about $P$ in light of the evidence or arguments presented to her, as opposed to simply taking another's word for $P$ being true. ${ }^{6}$ Since epistemic autonomy concerns only direct influence, being epistemically autonomous with regard to $P$ is perfectly compatible with forming and modifying one's opinions about $P$ in light of evidence and arguments presented by other people, including other experts.

Another important point is that epistemic autonomy is clearly a matter of degree. That is, one's attitudes can be more and less influenced by another persons' attitudes, roughly to the extent that one is likely to adopt the other person's attitudes. Later (in section 4) I will give a precise probabilistic explication of what this amounts to. For now, note that even if it is unrealistic or impossible for experts to be completely autonomous in the above sense, we can still ask whether having any degree of epistemic autonomy has epistemic value. What I will argue below is that, all other things being equal, even a very slight degree of autonomy has a distinct kind of epistemic value. Although this type of epistemic value can be trumped or outweighed by other considerations against being epistemically autonomous, such as by the value of reaching a consensus as quickly as possible, it's nevertheless true that there is something valuable about any increased degree of epistemic autonomy. Put differently,

\footnotetext{
${ }^{6}$ The distinction I am making here is similar, if not identical, to Gibbard's distinction between what he calls 'authority' and 'Socratic influence' (Gibbard, 1990, 174-5); it is even more similar, perhaps even identical, to Foley's distinction between 'authority' and 'influence' (Foley, 2001, 85).
} 
the argument will be that being more epistemically autonomous is pro tanto valuable.

Finally, note that epistemic autonomy is defined relative to a specific proposition $P$. We can generalize this definition slightly by also defining epistemic autonomy with respect to a domain of expertise $D$ as being epistemically autonomous with regard to all the propositions in, or relevant to, $D .{ }^{7}$ So, for example, a marine biologist might be said to be epistemically autonomous with regard to salmon hatching in Iceland if she is autonomous with regard to all propositions about the hatching of Icelandic salmon. Now, while it may be difficult or even impossible to be epistemically autonomous with regard to an unrestricted set of all propositions (even within some particular scientific field or subfield, such as marine biology), it is certainly not impossible to be epistemically autonomous with regard to a sufficiently restricted domain of expertise. In our example, it is surely possible for the marine biologist to remain at least partly uninfluenced (in a direct way) by her colleagues' opinions on Icelandic salmon hatching.

I conclude that it is not impossible for experts to be epistemically autonomous in the relevant sense of the term. However, even granting the possibility of experts exhibiting epistemic autonomy, one might still doubt that being autonomous would ever be epistemically rational. (This is the second challenge mentioned above.) Consider, in particular, Linda Zagzebski's argument that the ideal of epistemically autonomous belief-formation involves an 'egoistic' discrimination between one's own evaluation of $P$ and other people's evaluations of $P .{ }^{8}$ Zagzebski claims that this is incoherent for rational, truth-seeking agents:

... if the epistemic egoist [i.e., the epistemically autonomous agent]

\footnotetext{
${ }^{7}$ This conception of a domain of expertise is a bit rough, but precise enough for our purposes here. If we want to be more precise, we can simply define a domain of expertise either as a set of related propositions $P_{1}, \ldots, P_{k}$; or alternatively as an n-tuple one of whose elements is that set of propositions (where other elements might include, e.g., the methods used in the domain).

${ }^{8} \mathrm{~A}$ similar argument against the ideal of epistemic autonomy is given by Richard Foley (2001, esp. 99-107). For a slightly different argument for the same conclusion, see Fricker (2006). Epistemic autonomy should not be confused with what Zagzebski later calls 'intellectual autonomy' (Zagzebski, 2013).
} 
is rational, she is committed to trusting others when they are conscientious, when they have the qualities she trusts in herself. Trusting herself commits her to trusting others when they are in the same position she is in; that is, when they are in similar circumstances, have apparently similar powers and abilities, and act as conscientiously as she acts when she trusts herself. If she is consistent, she must trust them as much as herself, other things being equal, since she has no basis upon which to trust herself more than those she perceives to be epistemically equally well-placed (Zagzebski, 2007, 257).

Assuming that Zagzebski's argument is sound, it would seem to undermine any ideal of epistemic autonomy, including the ideal of epistemic autonomy for experts. ${ }^{9}$

Appearances perhaps to the contrary, however, an important form of the ideal of expert autonomy can be maintained even if one grants Zagzebski's antiegoistic argument. Zagzebski's argument is concerned with what we should believe on the basis of other people's testimony, and it is indeed plausible that one's beliefs should reflect the totality of one's evidence pertaining to a proposition - including one's testimonial evidence. However, note that at least sometimes when we ask experts for their opinion on a given issue, we are not asking them for what they believe all things considered, i.e. in light of all the evidence available to them (which includes their testimonial evidence).

\footnotetext{
${ }^{9}$ Many other epistemologists would seem to be implicitly committed to the denial of the ideal of epistemic autonomy for experts. Consider, in particular, the debate about peer disagreement. Conciliatory views of peer disagreement hold that, upon discovering that one disagrees about $P$ with someone who is equally intelligent and equally well-informed regarding $P$, one should modify one's opinion about $P$ by moving closer to the opinion of one's peer, i.e. by suspending belief or adopting an intermediate degree of belief in $P$ (see, e.g. Frances, 2005; Feldman, 2006; Christensen, 2007; Elga, 2007; Lackey, 2010; Matheson, 2015). Assuming that pairs of experts are at least sometimes equally intelligent and wellinformed with regard to some domain of expertise, it follows that experts should generally not be epistemically autonomous with regard to those domains. Indeed, it would seem that many non-conciliationists are implicitly committed to rejecting the ideal of epistemic autonomy for experts as well, since they often acknowledge that at least one of the parties to the disagreement (viz. the one whose belief is not in fact supported by the evidence) should modify her opinion on $P$ when realizing that her peer disagrees with her about $P$ (see, e.g., Kelly, 2005, 2010).
} 
Rather, we are (sometimes) eliciting their judgment of the extent to which some specific subset of that evidence - such as the outcome of specific a set of experiments, tests, or observations - supports a given theory or hypothesis. For example, a doctor who is asked to give a 'second opinion' may be asked to 'bracket' the other doctor's diagnosis for that purpose, reporting not what she believes all things considered, but on her own diagnosis based only on the patient's symptoms and test results (or some such suitably restricted subset of her evidence).

So the kinds of opinions we elicit from experts are not always their beliefs all things considered. To help us understand this, let me introduce a distinction between belief and acceptance along the lines suggested by Jonathan Cohen (1992). On Cohen's distinction, one believes that $P$ just in case one is disposed to feel it true that $P$ and false that $\neg P$. As Cohen notes, such a disposition is quite independent of "whether or not one is willing to act, speak, or reason accordingly" (Cohen, 1992, 4). By contrast, one accepts that $P$ just in case one has a policy of treating it as given that $P$ in a particular context, where treating a proposition as given involves "including that proposition ... among one's premisses for deciding what to do or think" in the context in question (Cohen, 1992, 4). In sum, then, while belief is a matter of what one is disposed to feel to be true, acceptance is a matter of what one has a policy to treat as true in a given context.

Belief and acceptance are both broadly-speaking epistemic attitudes, but they differ in two important respects. First, acceptance is a straightforwardly voluntary attitude, in the sense that whether we adopt a policy of treating something as true is under our control in a straightforward way. By contrast, if our beliefs are under voluntary control at all, it is certainly more difficult to adopt or reject beliefs at will. ${ }^{10}$ A second important difference between belief and acceptance is that the latter is sensitive to context in a way that the former

\footnotetext{
${ }^{10}$ That beliefs are involuntary in this way was influentially argued by Williams (1973). However, one need not assume that beliefs are completely outside of one's control in order for there to be a difference between acceptance and belief with respect to the extent to which they are voluntary or controlled, since acceptance would still be voluntary in a much more straightforward way. After all, there is no special cognitive effort required to adopt a policy of treating propositions as given in a particular context, while it would certainly require some special circumstances and/or abilities to believe a proposition at will.
} 
is not. Indeed, it is built into the concept of acceptance that one can accept a proposition - i.e. treat it as given - in some contexts without accepting it in all contexts. Thus, for example, our doctor may treat her own diagnosis as given in the context of providing her patient with a second opinion, even though she may not do so in other contexts (e.g. in discussions with her colleagues). By contrast, belief is not sensitive to context in the same way. After all, we would never say that the doctor's beliefs about the correct diagnosis suddenly changes when she starts to provide her second opinion. ${ }^{11}$ The point here is not that acceptance is context-sensitive whereas belief is not (though that might also be true); rather, the point is that acceptance is clearly context-sensitive in a different and much more straightforward way than belief.

Now, since acceptance is relative to a context in this way - one always treats something as true in a given context - we can distinguish between different kinds of acceptance depending on the context in which they occur. These 'contexts' are perhaps most clearly distinguishable when one explicitly takes on a specific institutional role that involves treating certain propositions as given. Our concern here is with what someone treats as true in the context of their individual pronouncements as experts in a given field - what we might call expert acceptance. Thus expert acceptance of a proposition $P$ amounts to having a policy of treating $P$ as given in the context of speaking as an individual expert in a domain that includes $P$. As we noted above in the case of a doctor's 'second opinion', one may offer something as her expert opinion even if one does not believe it to be true all things considered. Thus this case illustrates that it is possible for expert acceptance to come apart from belief.

This distinction between belief and expert acceptance allows us to distinguish two kinds of epistemic autonomy:

Epistemic autonomy $: S$ is epistemically autonomous $s_{B}$ with respect to a proposition $P$ to the extent that $S$ 's belief regarding $P$ is not directly influenced by other agents' beliefs regarding $P$.

\footnotetext{
${ }^{11}$ Of course, belief (or belief-attributions) may well be context-sensitive in other ways, e.g. by depending on the context of the person attributing belief in much the same way that knowledge-attributions are according to epistemic contextualism. See, for example, Cohen (1986, 2005), DeRose (1992), Lewis (1996), Neta (2002), and Conee (2005).
} 
Epistemic autonomy $_{\mathbf{A}}: S$ is epistemically autonomous a $_{A}$ with respect to a proposition $P$ to the extent that $S$ 's expert acceptance regarding $P$ is not directly influenced by other agents' expert acceptance regarding $P$.

This distinction between two different kinds of epistemic autonomy is important for present purposes because it opens up the possibility of accepting Zagzebski's anti-egoistic argument against autonomous believing - and more generally of rejecting the idea that believers should ever ignore or dismiss testimony from their epistemic equals or superiors - and at the same time recognizing a place for a different type of intellectual independence, viz. epis-

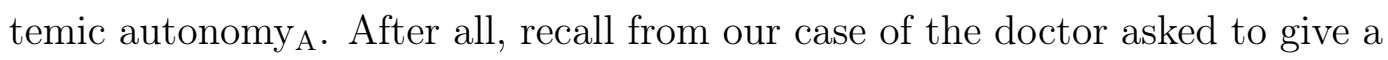
second opinion that there might be reasons for experts in particular to have or adopt certain epistemic ideals with regard to what they accept in the context of their work as experts that aren't also reasons for them to adopt analogous ideals with regard to their beliefs. ${ }^{12}$

Of course, I have not yet discussed any such reasons apart from the somewhat vague suggestion that experts should ideally not engage in 'groupthink' or exhibit 'herd mentality'. Fleshing out this suggestion is the task I have set myself below. But let me first be more precise about the sense in which I take epistemic autonomy $y_{\mathrm{A}}$ to be an ideal for experts:

The Ideal of Expert Autonomy: It is pro tanto valuable for experts to be (more) epistemically autonomous $\mathrm{A}_{\mathrm{A}}$ of other experts with regard to their mutual domain(s) of expertise.

Put differently, the claim here is that in groups of experts on the same domain, there is a pro tanto value to being more rather than less epistemically autonomous $_{\mathrm{A}}$, where a pro tanto value is a (not merely prima facie) value

\footnotetext{
${ }^{12}$ More generally, the idea here is that those who occupy certain roles might be subject to specific epistemic ideals in what they treat as given in the context of occupying those roles. This idea should be familiar from the legal domain, e.g. from the way in which jurors are required to disregard hearsay and illegally obtained evidence in reaching their verdict, even when such evidence would (and arguably should) influence their private beliefs. Similarly, it seems at least plausible that methodological norms in various sciences require scientific acceptance of hypotheses to be based on particular kinds of systematic empirical data and statistical analysis, rather than, say, on anecdotal and testimonial evidence of various kinds. (Thanks to an anonymous reviewer for suggesting this analogy.)
} 
that may be trumped or outweighed by other considerations. Roughly, this amounts to the claim that there is always something valuable about experts being epistemically autonomous ${ }_{A}$ to a greater extent, even when this value has less weight than countervailing considerations. ${ }^{13}$

Now, my main concern below is with providing a rational basis - a rationale - for this ideal. What, if anything, makes the Ideal of Expert Autonomy true? Note that the answer cannot be that being epistemically autonomous makes each expert in a group individually more reliable, for this is precisely what we cannot assume in cases when one expert is considering whether to be directly influenced by the opinions of her fellow experts. Since the expert in question is assumed to be no more of an expert than her fellow experts, she will be at least as likely to accept the truth if she blindly trusts her colleagues as if she makes up her mind independently. To be more precise, then, the question is how there could be this ideal of expert autonomy given that being epistemically autonomous does not increase the individual reliability of such experts. In what follows, I propose to answer this question by appealing to the effects of epistemic autonomy at the social level of groups of experts with the same domain of expertise. Specifically, I suggest that the value of expert autonomy lies in the fact that groups of epistemically autonomous agents can provide us with an especially reliable kind of testimonial evidence. I will refer to this as the Whewellian Rationale.

\section{Expert Autonomy and Testimonial Consilience}

\subsection{The Whewellian Rationale}

William Whewell coined the term 'consilience of inductions' to describe cases in which a theory receives support from unrelated pieces of evidence, as opposed to being supported by evidence that is similar or closely related. Whewell argued that this amounts to an especially powerful type of inductive evidence

\footnotetext{
${ }^{13}$ Since I will henceforth only be concerned with epistemic autonomy ${ }_{A}$ (as opposed to autonomy $_{\mathrm{B}}$ ), I will often drop the subscript 'A' from now on, e.g. writing 'epistemically autonomous' instead of 'epistemically autonomous ${ }_{\mathrm{A}}$ '. I will also frequently use 'expert autonomy' as a shorthand for 'epistemic autonomy among experts'.
} 
in science:

That rules springing from remote and unconnected quarters should thus leap to the same point, can only arise from that being the point where the truth resides. [...] Accordingly the cases in which inductions from classes of facts altogether different have thus jumped together, belong only to the best established theories which the history of science contains. And as I shall have occasion to refer to this peculiar feature of their evidence, I will take the liberty of describing it by a particular phrase; and will term it the Consilience of Inductions (Whewell, 1858, 88).

Whewell's point is not just that a theory is better confirmed if more evidence speaks in favor of it; rather, Whewell is suggesting that, other things being equal, a theory is better confirmed if the pieces of evidence that support a theory are independent of each other.

Now, what's true for evidence generally is also true of testimonial evidence. If Whewell is right that evidence from independent sources provides stronger support for theories than otherwise similar evidence from dependent (or less independent) sources, then a claim accepted on the basis of independent pieces of testimony should provide stronger support than otherwise similar, but dependent, pieces of testimony. In other words, Whewell's general point about the justificatory power of consilient evidence implies that we have more reason to accept what a number of independent agents assert than an otherwise identical groups of agents whose testimony is dependent in some way. This is relevant to the Ideal of Expert Autonomy since epistemic autonomy is effectively a kind of testimonial independence between agents. Thus Whewell's point about consilience suggests that epistemic autonomy ensures a kind of independence between agents that makes their joint testimony a more reliable guide to truth than it would otherwise be.

In this Whewellian spirit, I want to suggest that the value of expert autonomy lies in the fact that an agreement in the testimony of scientific experts is a more reliable guide to truth when the experts are epistemically autonomous. This reliability of agreement in expert testimony is in turn valuable because of its beneficial epistemic effects on those who are not experts on the particular 
issue in question. In particular, it enables experts in other domains to rely on such testimony in their own research, which promotes cumulative intellectual progress; similarly, it enables laypeople to safely rely on agreement in expert testimony in applying the results of experts' investigations, e.g. in technology and policy-making, which increases the applicability of these results. In sum:

The Whewellian Rationale: Expert autonomy is pro tanto valuable because, and in so far as, (W1) agreement in expert testimony is more reliable when experts are more epistemically autonomous $\mathrm{A}_{\mathrm{A}}$, and (W2) increased reliability of such agreement promotes cumulative intellectual progress and safe applications of expert inquiry.

I have separated the Whewellian Rationale into two separate claims, (W1) and (W2), because I will argue for each claim separately (in $\S 3.2$ and $\S 3.3$ respectively).

Before I do that, however, let me emphasize that the Whewellian Rationale locates only a pro tanto value in expert autonomy. As such, this value will have to be weighed against other considerations in determining whether expert autonomy is valuable all things considered. In particular, it is plausible that increasing epistemic autonomy among experts would reduce the amount of agreement among them and/or slow down the process of them reaching agreement. If so, the value located by the Whewellian Rationale could be outweighed by the costs of having fewer hypotheses on which the experts agree at a given time. This is not an objection to the Whewellian Rationale, since it does not purport to show that the value of expert autonomy cannot be outweighed by other considerations. One might of course wonder whether there is a way of determining exactly under what circumstances the pro tanto value of expert autonomy would be outweighed in this way. While this would be a worthwhile task, I will not offer any suggestions for how to do it below. Thus, as far as this paper is concerned, we will have to rest content with showing that expert autonomy is pro tanto valuable, regardless of the circumstances under which it is also valuable all things considered. 


\subsection{How Expert Autonomy Enhances Joint Reliability}

(W1) in effect holds that agreement between epistemically autonomous experts is a more reliable indicator of truth than agreement between less autonomous, but otherwise identical, experts. Since agreement between epistemically autonomous experts amounts to a kind of testimonial consilience, which is a special case of what is arguably an especially powerful type of inductive evidence, (W1) already has considerable prima facie plausibility. This section bolsters this intuition by providing a rigorous probabilistic argument for (W1). The argument proceeds by comparing the probability that a hypothesis $H$ is true given that a group of (somewhat) autonomous agents all attest to its truth, with the probability that $H$ is true given that an otherwise identical group of less autonomous agents do the same. As we shall see, it can be shown that the probability for $H$ is higher given agreement among more autonomous experts than given agreement among their less autonomous counterparts.

To set up the argument, the first thing to do is to find a way to represent in a probabilistic framework the assumption that one group of experts exhibits more epistemic autonomy than another group. ${ }^{14}$ Recall that epistemic autonomy $_{\mathrm{A}}$ with regard to some proposition $H$ is defined in terms of the extent to which an agent's acceptance of $H$ is not directly influenced by another's acceptance of $H$. This direct influence of one agent's acceptance of $H$ on another's translates straightforwardly into a probabilistic framework in terms of the extent to which one agent's acceptance of $H$ is positively dependent on the other agent's acceptance of $H$. In probability theory, a collection of $n$

\footnotetext{
${ }^{14}$ The probabilistic conception of epistemic autonomy that I propose below is closely analogous to a measure of evidential independence that was informally proposed by Howson and Urbach $(1989,114)$, and later formalized and generalized by Wayne $(1995,112-113)$ and Myrvold (1996, 661-662) (for discussion, see e.g. Fitelson, 2001; Bovens and Hartmann, 2003; Wheeler, 2009; Claveau, 2013; McGrew, 2016) and to Shogenji's (1999, 338-340) measure of coherence among propositions (for discussion, see e.g. Akiba, 2000; Shogenji, 2001; Fitelson, 2003; Olsson, 2005; Schupbach, 2008, 2011). Of course, I am interested here not in the adequacy of these measure of evidential independence and coherence, but in whether an analogous measure can be used to account for the value of epistemic autonomy. Although the literatures on these measures are voluminous, I have not been able to find analogues of the results discussed below for epistemic autonomy. (Another natural place to look for analogous results is the literature on the Condorcet Jury Theorem (CJT). Footnote 18 explains why the CJT is not in fact suitable for our purposes here.)
} 
propositions, $P_{1}, \ldots, P_{n}$, are said to be (positively) dependent just in case the probability of their conjunction is greater than the product of their individual probabilities, i.e. just in case:

$$
\operatorname{Pr}\left(\bigwedge_{i=1}^{n} P_{i}\right)>\prod_{i=1}^{n} \operatorname{Pr}\left(P_{i}\right)
$$

This is a binary, as opposed to graded, notion of probabilistic dependence - it tells us when some propositions exhibit any probabilistic dependence at all, but it doesn't tell us how much dependence there is between them. Since epistemic autonomy admits of degrees (cf. section 2), we need a graded, rather than merely a binary, notion of dependence. Fortunately, however, we can define a graded notion of dependence, $\operatorname{Dep}\left(P_{1}, \ldots, P_{n}\right)$, as the ratio of the left-hand side of (1) to its right-hand side:

$$
\operatorname{Dep}\left(P_{1}, \ldots, P_{n}\right)=\frac{\operatorname{Pr}\left(\bigwedge_{i=1}^{n} P_{i}\right)}{\prod_{i=1}^{n} \operatorname{Pr}\left(P_{i}\right)}
$$

We can use this graded notion of dependence to compare how much probabilistic dependence there is in two collections of propositions of equal size. ${ }^{15}$

The idea, then, is to represent the relative epistemic autonomy of two otherwise identical groups of experts in terms of the degree to which their acceptance of $H$ is dependent on each other's acceptance - where a greater degree of dependence corresponds to a lesser degree of autonomy. Thus, where ' $X_{i}(H, E)$ ' denotes the proposition that an expert $X_{i}$ with evidence $E$ accepts $H$, we say that a group of experts $X_{1}^{A}, \ldots, X_{n}^{A}$ exhibits more epistemic autonomy with respect to $H$ and $E$ than an otherwise similar group of experts $X_{1}^{B}, \ldots, X_{n}^{B}$ just in case:

$$
\operatorname{Dep}\left(X_{1}^{A}(H, E), \ldots, X_{n}^{A}(H, E)\right)<\operatorname{Dep}\left(X_{1}^{B}(H, E), \ldots, X_{n}^{B}(H, E)\right)
$$

\footnotetext{
${ }^{15}$ Notice that we can use our graded notion of dependence to define binary notions of dependence and independence: $P_{1}, \ldots, P_{n}$ are positively dependent iff $\operatorname{Dep}\left(P_{1}, \ldots, P_{n}\right)>1$, negatively dependent iff $\operatorname{Dep}\left(P_{1}, \ldots, P_{n}\right)<1$, and (mutually) independent otherwise. Thus we obtain (1) as a special case.
} 
Now, since this explication of comparative degrees of expert autonomy appeals to probabilities, the question arises how these probabilities should be interpreted. So far we have been concerned with explicating what it is for groups of experts to really be more and less autonomous, so these probabilities must be interpreted as referring to something more objective than a particular rational agent's subjective probability assignments, e.g. objective chances or frequencies. However, in so far as a particular rational agent is aware that the relevant objective chances or frequencies satisfy the inequality in (3), the principal principle (in case of objective chances) or the principle of direct inference (in the case of frequencies) ensures that the agent's subjective credences satisfy (3) as well. Thus, in what follows, I will assume that a rational agent's subjective probability assignments satisfy this condition - and, more generally, that such an agent's probability assignments reflect her knowledge of objective chances and frequencies. ${ }^{16}$

What I will seek to show is that, for such a rational agent, agreement among the more autonomous experts $X_{1}^{A}, \ldots, X_{n}^{A}$ is, other things being equal, a greater indicator of the truth than agreement among their less autonomous counterparts, $X_{1}^{B}, \ldots, X_{n}^{B}$. So, suppose first that other things are indeed equal; in particular, suppose that our agent is aware that, given that $H$ is true, the two groups of experts are equally likely to agree that $H$ is true:

$$
\operatorname{Pr}\left(\bigwedge_{i=1}^{n} X_{i}^{A}(H, E) \mid H\right)=\operatorname{Pr}\left(\bigwedge_{i=1}^{n} X_{i}^{B}(H, E) \mid H\right)
$$

Suppose also that our agent is aware that, individually, the corresponding experts in each group are equally likely to accept $H$ in light of $E$ :

$$
\operatorname{Pr}\left(X_{i}^{A}(H, E)\right)=\operatorname{Pr}\left(X_{i}^{B}(H, E)\right), \text { for } 1 \leq i \leq n .
$$

\footnotetext{
${ }^{16}$ I will also assume in what follows that all propositions have positive probability (which ensures that $\operatorname{Dep}\left(P_{1}, \ldots, P_{n}\right)$ is always well-defined). Since rationality is often taken to prohibit assignments of extreme probabilities (i.e. probabilities zero and one), this assumption can be thought of as built into the requirement that the relevant agent is rational; alternatively, this assumption can be viewed as a restriction of the argument given below - leaving it as an open question whether the argument can be extended to notions of dependence that allow for zero-probability events.
} 
Under these ceteris paribus conditions, it can be shown that (3) entails:

$$
\operatorname{Pr}\left(H \mid \bigwedge_{i=1}^{n} X_{i}^{A}(H, E)\right)>\operatorname{Pr}\left(H \mid \bigwedge_{i=1}^{n} X_{i}^{B}(H, E)\right)
$$

I.e., the probability that $H$ is true is greater given an agreement among the more autonomous experts $X_{1}^{A}, \ldots, X_{n}^{A}$ than it is given an agreement among their less autonomous counterparts $X_{1}^{B}, \ldots, X_{n}^{B} \cdot{ }^{17}$ In sum, then, if two pairs of respectively more and less autonomous experts are otherwise identical in relevant respects - in that the two groups are equally likely to agree on the truth, and each expert is individually equally likely to accept a given hypothesis $H$ as the expert's counterpart in the other group - then agreement on $H$ among

${ }^{17}$ Proof: We prove that (3), (4), and (5) jointly entail (6). First note that by our definition of graded dependence, (3) is equivalent to:

$$
\frac{\operatorname{Pr}\left(\bigwedge_{i=1}^{n} X_{i}^{A}(H, E)\right)}{\prod_{i=1}^{n} \operatorname{Pr}\left(X_{i}^{A}(H, E)\right)}<\frac{\operatorname{Pr}\left(\bigwedge_{i=1}^{n} X_{i}^{B}(H, E)\right)}{\prod_{i=1}^{n} \operatorname{Pr}\left(X_{i}^{B}(H, E)\right)}
$$

Given (5), the denominators on each side of this are equal, so we have:

$$
\operatorname{Pr}\left(\bigwedge_{i=1}^{n} X_{i}^{A}(H, E)\right)<\operatorname{Pr}\left(\bigwedge_{i=1}^{n} X_{i}^{B}(H, E)\right)
$$

Since $\operatorname{Pr}(H) \neq 0$ (see footnote 16 ), we preserve the inequality when multiplying both sides with $\operatorname{Pr}(H)$ :

$$
\operatorname{Pr}(H) \times \operatorname{Pr}\left(\bigwedge_{i=1}^{n} X_{i}^{A}(H, E)\right)<\operatorname{Pr}(H) \times \operatorname{Pr}\left(\bigwedge_{i=1}^{n} X_{i}^{B}(H, E)\right)
$$

Rearranging, we get:

$$
\frac{\operatorname{Pr}(H)}{\operatorname{Pr}\left(\bigwedge_{i=1}^{n} X_{i}^{A}(H, E)\right)}>\frac{\operatorname{Pr}(H)}{\operatorname{Pr}\left(\bigwedge_{i=1}^{n} X_{i}^{B}(H, E)\right)}
$$

Given (4), we preserve the inequality when multiplying each side with the corresponding side of (4), obtaining:

$$
\frac{\operatorname{Pr}(H) \times \operatorname{Pr}\left(\bigwedge_{i=1}^{n} X_{i}^{A}(H, E) \mid H\right)}{\operatorname{Pr}\left(\bigwedge_{i=1}^{n} X_{i}^{A}(H, E)\right)}>\frac{\operatorname{Pr}(H) \times \operatorname{Pr}\left(\bigwedge_{i=1}^{n} X_{i}^{B}(H, E) \mid H\right)}{\operatorname{Pr}\left(\bigwedge_{i=1}^{n} X_{i}^{B}(H, E)\right)}
$$

By Bayes's Theorem, this is equivalent to (6). 
the autonomous experts is a more reliable guide to $H$ 's truth. In this sense, epistemic autonomy among experts increases the probability that agreed-upon theories are true, other things being equal. ${ }^{18}$

One might of course question whether the two ceteris paribus conditions - i.e., equations (4) and (5) - describe features that can and should be held constant in a comparison between groups with different degrees of epistemic autonomy. Let's consider these conditions in reverse order. Equation (5) requires that, taken one by one, the individual members of the two groups are exactly comparable with regard to how likely they are to accept $H$ regardless of its truth (i.e. not conditional on $H$ ). Somewhat more precisely, it requires that the members of each group can be paired, one-to-one, with the members of the other group in such a way that the paired agents be equally likely to accept $H$ regardless of whether it is true. As I interpret this condition, it requires that any bias for or against accepting $H$ held by an agent in one of

\footnotetext{
${ }^{18}$ This result may remind some readers of the idea, often associated with Condorcet's Jury Theorem (CJT), that under certain conditions a group of independent and individually competent agents is more likely to be correct in a majority vote than any of the group's proper subsets. One might even think that the argument above implicitly appeals to the CJT. To see why, consider an informal statement of the theorem:
}

\section{Condorcet Jury Theorem (CJT): If}

(a) each member of a group is more likely than not to reach a correct conclusion (Competence), and

(b) the probabilities of each member reaching a correct conclusion are independent (Independence),

then the probability that the majority has reached a correct conclusion (i) increases with the size of the group, and (ii) converges to one as the group's size goes to infinity.

Condition (b) - Independence - might seem close enough to what I have called 'epistemic autonomy', so it might seem as if the CJT shows how epistemic autonomy increases group reliability in the relevant way. (Something like this suggestion is made in a different context by Hazlett $(2016,134-7)$.)

In fact, however, the CJT is not suitable as part of an argument for (W1). One immediate problem is that Independence is an extremely demanding (binary) condition, which effectively applies only when there is no influence at all between the agents in a given group. This by itself is not a major problem, for there are generalizations of the CJT which do apply for lesser degrees of independence (e.g., Boland, 1989; Boland et al., 1989; Berg, 1993). The more serious problem is that the CJT tells us about the reliability of a group as a function of the group's size (given Competence and Independence, or some fixed degrees thereof), but we are not interested in how reliability varies with size of the group. Instead, we are concerned with how reliability varies with degrees of epistemic autonomy, i.e. degrees of 'independence'. Since this factor is a constant in the CJT, the theorem simply doesn't tell us what we want to know. 
the groups is matched by a corresponding bias in an agent in the the other group. This is clearly something that can be held constant even when the two groups exhibit different degrees of epistemic autonomy, since the epistemic autonomy exhibited by a group is not a matter of how biased or unbiased each of its members are regarding $H$. Furthermore, note that if this condition is violated, we would be comparing groups with different individual biases regarding $H$, which could easily translate into a difference in the likelihoods that all the members of each group accept $H$. Thus this is exactly the type of factor that ought to be held constant as we compare what a consensus in groups with more versus less epistemic autonomy tells us about whether $H$ is likely to be true.

Next consider equation (4). It requires that, conditional on $H$ being true, the two groups of experts are equally likely to agree that $H$ is true. My reason for including this as a ceteris paribus condition is that if one of the group is more likely to agree that $H$ is true when $H$ is indeed true, then this would already favor that group's consensus over the other group's as an indicator of the truth regarding $H$ - regardless of the degree to which the two groups are epistemically autonomous. For example, one way in which (4) could fail is if one of the groups was simply more knowledgeable about the sorts of issues that arise regarding how to evaluate $H$ (e.g. because they have more experience of evaluating similar hypotheses). This is clearly the sort of factor that we want to hold fixed in a comparison of how reliable a guide to truth an agreement in groups of more versus less autonomous experts would be. Hence it certainly seems desirable to impose the restriction described by equation (4) in our comparison of more and less epistemically autonomous groups of experts.

One might still worry that this requirement is implausible or rarely satisfied for groups of agents that exhibit different degrees of epistemic autonomy. If so, the epistemic value of expert autonomy might seem to be based on an unrealistic idealization. It is thus of interest to note that (6) can also be 
derived without (4) given the following condition:

$$
\frac{\operatorname{Dep}\left(X_{1}^{B}(H, E), \ldots, X_{n}^{B}(H, E)\right)}{\operatorname{Dep}\left(X_{1}^{A}(H, E), \ldots, X_{n}^{A}(H, E)\right)}>\frac{\operatorname{Pr}\left(\bigwedge_{i=1}^{n} X_{i}^{B}(H, E) \mid H\right)}{\operatorname{Pr}\left(\bigwedge_{i=1}^{n} X_{i}^{A}(H, E) \mid H\right)}
$$

In other words, (4) can be replaced with the condition that the ratio of dependence in the more versus less autonomous group must be greater than the ratio of their corresponding likelihoods of agreeing on $H$, conditional on $H$ being true. ${ }^{19}$ This additional result tells us that even when the likelihoods of the two groups agreeing on $H$ given that $H$ is true are unequal - e.g. because the less autonomous group is simply more knowledgeable regarding $H$ - this can be outweighed by a corresponding difference in the degree of epistemic autonomy exhibited by the two groups. Put differently, a consensus among a more autonomous group of experts would be a more reliable guide to truth even when such a group is less likely to agree on the truth than a corresponding group of less autonomous experts, provided that the former's degree of autonomy exceeds that of the latter's to a sufficiently high degree.

${ }^{19}$ Proof: We prove that (5) and (7) jointly entail (6). Given our definition in (2), (7) can be written as follows:

$$
\frac{\operatorname{Pr}\left(\bigwedge_{i=1}^{n} X_{i}^{B}(H, E)\right) / \prod_{i=1}^{n} \operatorname{Pr}\left(X_{i}^{B}(H, E)\right)}{\operatorname{Pr}\left(\bigwedge_{i=1}^{n} X_{i}^{A}(H, E)\right) / \prod_{i=1}^{n} \operatorname{Pr}\left(X_{i}^{A}(H, E)\right)}>\frac{\operatorname{Pr}\left(\bigwedge_{i=1}^{n} X_{i}^{B}(H, E) \mid H\right)}{\operatorname{Pr}\left(\bigwedge_{i=1}^{n} X_{i}^{A}(H, E) \mid H\right)}
$$

By (5), this simplifies to:

$$
\frac{\operatorname{Pr}\left(\bigwedge_{i=1}^{n} X_{i}^{B}(H, E)\right)}{\operatorname{Pr}\left(\bigwedge_{i=1}^{n} X_{i}^{A}(H, E)\right)}>\frac{\operatorname{Pr}\left(\bigwedge_{i=1}^{n} X_{i}^{B}(H, E) \mid H\right)}{\operatorname{Pr}\left(\bigwedge_{i=1}^{n} X_{i}^{A}(H, E) \mid H\right)}
$$

Rearranging and multiplying both sides by $\operatorname{Pr}(H)$, we get:

$$
\frac{\operatorname{Pr}(H) \times \operatorname{Pr}\left(\bigwedge_{i=1}^{n} X_{i}^{A}(H, E) \mid H\right)}{\operatorname{Pr}\left(\bigwedge_{i=1}^{n} X_{i}^{A}(H, E)\right)}>\frac{\operatorname{Pr}(H) \times \operatorname{Pr}\left(\bigwedge_{i=1}^{n} X_{i}^{B}(H, E) \mid H\right)}{\operatorname{Pr}\left(\bigwedge_{i=1}^{n} X_{i}^{B}(H, E)\right)}
$$

Which is equivalent to (6) by Bayes's Theorem. 


\subsection{What Good is Reliability of Expert Agreement?}

Having seen how epistemic autonomy increases the reliability of expert agreement via a kind of testimonial consilience, the next thing to consider is why this kind of reliability is something worth having in expert groups. Clearly, the answer will have something to do with the fact that we look to experts to provide us with correct information about issues falling within their domain of expertise. But it is worth digging a little deeper here, asking ourselves why it's so important that the claims on which experts within some domain are in agreement can reliably be taken as true. As alluded to before, I want to suggest that there are two importantly different considerations here.

On the one hand, reliability of expert agreement is crucial to laypeople who rely on expert opinion as a source of information. Consider, for example, the way in which scientific results communicated by experts are used in policy making processes, including public policy. In contexts of that sort, agreement among experts must be taken to be reliable in order for their testimony to serve its purpose as a basis for policy. For example, consider the frequentlycited consensus on anthropogenic climate change - the theory that human influence is a significant contributing cause of the recent rise in mean global temperatures. Reliability of expert agreement is valuable, in part, because we want to be able to cite expert agreements of this kind in arguments that the agreed-upon theories are indeed true (or likely to be true). If agreement among experts were not a reliable guide to truth, scientifically-informed public policy would be more-or-less impossible in its current form.

On the other hand, reliability of expert agreement is also valuable to other experts, i.e. experts whose domain of expertise does not overlap with that of the agreeing experts. Increasing specialization in nearly all fields of inquiry makes it impossible for anyone to be an expert outside a relatively limited domain. Thus, any expert in some domain $D_{i}$ will be a non-expert with regard to some other domain $D_{j}$. At the same time, results from the other domain $D_{j}$ will often be highly relevant to the expert's work in her own domain $D_{i}$,

\footnotetext{
${ }^{20}$ This is a familiar point. See, e.g., Hardwig $(1985,1991)$, Thagard (1997), Wray (2002), and Andersen and Wagenknecht (2013).
} 
such that our $D_{i}$-expert is in many ways forced to rely on the testimony of $D_{j^{-}}$ experts in order to make progress in her own investigations. ${ }^{20}$ In so far as our $D_{i}$-expert seeks correct information about $D_{j}$, she will rely only on what $D_{j^{-}}$ experts agree on; but of course that will only be reliable information in so far this kind of expert agreement is a reliable indicator of truth. So, a $D_{i \text {-expert }}$ can only trust information from $D_{j}$-experts in so far as agreement among the latter is a reliable indicator of truth. Since relying on experts' testimony in this way is indispensable to almost all contemporary inquiry, reliability of expert agreement is vital for cumulative intellectual progress.

It is worth emphasizing just how common it is for experts in different domains to rely on each other's work in this way, and thus how important it is for intellectual progress that groups of experts provide reliable testimony. Consider, for example, a theoretical astrophysicists who specializes in cosmic rays, high-energy radiation which originates outside of the Earth's atmosphere. Being a theoretical astrophysicist, she will not herself have made the empirical observations or constructed the data models on the basis of which her theories are constructed. Instead, she will rely on information on which there is agreement among her colleagues in observational astrophysics. Similarly, since cosmic rays are thought to consist of photons and other high-energy particles, theoretical research into cosmic rays frequently appeal to recent research in particle physics. However, while astrophysicists will be knowledgable about large-scale physical theories such as the general relativity, they are rarely experts on recent developments within particle physics, ${ }^{21}$ so they are typically forced to rely on what other experts agree on when their own research appeals to specific results about high-energy particles. Finally, like almost all other natural scientists, astrophysicists frequently rely on the expert opinions of mathematicians for theorems and derivations relevant to their own research.

In sum, then, reliability of expert agreement is valuable in at least two ways, viz. in (a) undergirding reliable application of expert knowledge, e.g. in public policy, and (b) promoting intellectual progress through reliable inter-domain

\footnotetext{
${ }^{21}$ Although there are notable exceptions, such as Samuel Ting at Princeton. Ting received the Nobel prize in physics for work on particle physics in 1976, but now leads a massive research project on cosmic rays (The Alpha Magnetic Spectrometer, AMS-02).
} 
communication between experts. Given the probabilistic argument presented earlier, this confers value on expert autonomy since agreement among more autonomous experts is a more reliable indicator of truth than agreement among less autonomous, but otherwise identical, experts. In this way, epistemic autonomy has a kind of instrumental value as a means to promoting reliable applications of expert knowledge and cumulative intellectual progress.

\section{Conclusion}

I began with the observation that there seems to be a sense in which, ideally, experts should make up their own minds about issues that fall within their domain of expertise, as opposed to following the opinions of their fellow experts. Partly in response to Zagzebski's anti-egoistic argument that privileging one's own belief-forming mechanisms would be incoherent, I provided an interpretation of this idea by appealing to the notion of expert acceptance - the Ideal of Expert Autonomy. I then argued that, all other things being equal, this kind of epistemic autonomy among experts makes their joint testimony more reliable, which in turn brings epistemic benefits both to laypeople and to experts in other fields - the Whewellian Rationale. In closing, it is worth drawing out how this support the general idea, endorsed by many social epistemologists, that the epistemic prescriptions appropriate for individuals may not serve the epistemic purposes of the groups such individuals comprise. ${ }^{22}$ According to the Whewellian Rationale, the value of epistemic autonomy does not consist in its being epistemically beneficial for the individual autonomous experts themselves. Rather, the value of this kind of autonomy is distinctively social in two distinct senses of the term: First, the epistemic benefits of expert

\footnotetext{
${ }^{22} \mathrm{~A}$ systematic discussion of this idea is provided by Mayo-Wilson, Zollman and Danks, who refer to it as the Independence Thesis (Mayo-Wilson et al., 2011, 654). Another wellknown argument for something like this idea is provided by Kitcher $(1990,1993)$ and Strevens (2003), who argue that a scientific community as a whole is more likely to discover truths when each scientist in it strives only for personal credit, rather than aiming to discover the truth. Similarly, Zollman $(2007,2010)$ argues that under certain conditions, groups of individuals who ignore certain information from other members will be more reliable than groups in which such information flows freely. Finally, Bishop (2005) argues that the respective principles of social and individual rationality recommend distinct, and indeed inconsistent, beliefs.
} 
autonomy are primarily conferred upon agents other than the autonomous experts themselves - viz. laypeople and other experts. Second, the epistemic value of expert autonomy is also social in the sense that these benefits arise not from the practice of consulting individual experts, but from that of consulting groups of experts about what its members agree on. ${ }^{23}$

\footnotetext{
${ }^{23}$ Research for this paper was supported by an Irish Research Council New Horizons grant for the project When Experts Disagree (Project ID: REPRO/2015/89). For discussions and helpful feedback, I am grateful to Maria Baghramian, Luke Drury, Elmar Unnsteinsson, Nick Hughes, Ed Nettel, MA students in the Disagreement module at University College Dublin, and audiences at the Third Lisbon International Conference on Philosophy of Science, Queens University Belfast, University College Dublin, and the University of Iceland. I am also indebted to Liam Kofi Bright, Elanor Taylor, Huginn Thorsteinsson, and an anonymous reviewer for Philosophy and Phenomenological Research for extraordinarily helpful comments on earlier versions of this paper.
} 


\section{REFERENCES}

Akiba, K. (2000). Shogenji's probabilistic measure of coherence is incoherent. Analysis, 60:356-359.

Andersen, H. and Wagenknecht, S. (2013). Epistemic dependence in interdisciplinary groups. Synthese, 190:1881-1898.

Berg, S. (1993). Condorcet's jury theorem, dependency among jurors. Social Choice and Welfare, 10:87-95.

Bishop, M. A. (2005). The Autonomy of Social Epistemology. Episteme, 2:6578.

Boland, P. J. (1989). Majority systems and the Condorcet jury theorem. The Statistician, 38:181-189.

Boland, P. J., Proschan, F., and Tong, Y. L. (1989). Modelling dependence in simple and indirect majority systems. Journal of Applied Probability, 26:81-88.

Bovens, L. and Hartmann, S. (2003). Bayesian Epistemology. Oxford University Press, Oxford.

Christensen, D. (2007). The Epistemology of Disagreement: The Good News. Philosophical Review, 116:187-218.

Claveau, F. (2013). The independence condition in the variety-of-evidence thesis. Philosophy of Science, 80:94-118.

Coady, C. A. J. (1992). Testimony: A Philosophical Study. Oxford University Press, Oxford.

Coady, D. (2006). When Experts Disagree. Episteme, 3:68-79.

Cohen, L. J. (1992). An Essay on Belief and Acceptance. Clarendon Press, Oxford.

Cohen, S. (1986). Knowledge and Context. Journal of Philosophy, 83:574-583.

Cohen, S. (2005). Contextualism Defended. In Steup, M. and Sosa, E., editors, Contemporary Debates in Epistemology, pages 56-62. Blackwell, Malden, 
MA.

Collins, H. and Evans, R. (2007). Rethinking Expertise. Chicago University Press, Chicago.

Conee, E. (2005). Contextualism Contested. In Steup, M. and Sosa, E., editors, Contemporary Debates in Epistemology, pages 47-56. Blackwell, Malden, MA.

DeRose, K. (1992). Contextualism and Knowledge Attributions. Philosophy and Phenomenological Research, 52:913-929.

Descartes, R. (1985/1628). Rules for the Direction of the Mind. In Cottingham, J., Stoothoff, R., and Murdoch, D., editors, The Philosophical Writings of Descartes, volume I. Cambridge University Press, Cambridge.

Elga, A. (2007). Reflection and Disagreement. Nous, 41:478-502.

Feldman, R. (2006). Reasonable Religious Disagreements. In Antony, L. M., editor, Philosophers Without Gods: Meditations on Atheism and the Secular Life, pages 194-214. Oxford University Press, Oxford.

Fitelson, B. (2001). A Bayesian Account of Independent Evidence with Applications. Philosophy of Science, 68:S123-S140.

Fitelson, B. (2003). A probabilistic theory of coherence. Analysis, 63:194-199. Foley, R. (2001). Intellectual Trust in Oneself and Others. Cambridge University Press, Cambridge.

Frances, B. (2005). Scepticism Comes Alive. Oxford University Press, Oxford.

Fricker, E. (1994). Against Gullibility. In Matilal, B. K. and Chakrabarti, A., editors, Knowing from Words, pages 125-161. Kluwer Academic Publishers, Boston.

Fricker, E. (2006). Testimony and Epistemic Autonomy. In Lackey, J. and Sosa, E., editors, The Epistemology of Testimony, pages 225-251. Oxford University Press, Oxford.

Gibbard, A. (1990). Wise Choices, Apt Feelings. Harvard University Press, Cambridge, Mass. 
Goldman, A. I. (2001). Experts: Which Ones Should You Trust? Philosophy and Phenomenological Research, 63:85-110.

Hardwig, J. (1985). Epistemic Dependence. The Journal of Philosophy, 82:335-349.

Hardwig, J. (1991). The Role of Trust in Knowledge. The Journal of Philosophy, 88:693-708.

Hazlett, A. (2016). The Social Value of Non-Deferential Belief. Australasian Journal of Philosophy, 94:131-151.

Howson, C. and Urbach, P. (1989). Scientific Reasoning: The Bayesian Approach. Open Court, La Salle, IL, 1st edition.

Kant, I. (1991/1784). An Answer to the Question: What is Enlightenment? In Reiss, H., editor, Political Writings, pages 54-60. Cambridge University Press, Cambridge, 2nd edition.

Kelly, T. (2005). The Epistemic Significance of Disagreement. In Gendler, T. and Hawthorne, J., editors, Oxford Studies in Epistemology, vol. 1, pages 167-196. Oxford University Press, Oxford.

Kelly, T. (2010). Peer Disagreement and Higher Order Evidence. In Feldman, R. and Warfield, T., editors, Disagreement, pages 111-174. Oxford University Press, Oxford.

Kitcher, P. (1990). The Division of Cognitive Labor. Journal of Philosophy, $87: 5-21$.

Kitcher, P. (1993). The Advancement of Science: Science without Legend, Objectivity without Illusions. Oxford University Press, New York.

Lackey, J. (2010). A Justificationalist View of Disagreement's Epistemic Significance. In Haddock, A., Millar, A., and Pritchard, D., editors, Social Epistemology, pages 298-325. Oxford University Press, Oxford.

Lane, M. (2014). When the experts are uncertain: Scientific knowledge and the ethics of democratic judgment. Episteme, 11:97-118.

Lewis, D. (1996). Elusive knowledge. Australasian Journal of Philosophy, 
$74: 549-567$.

Locke, J. (1975/1689). An Essay Concerning Human Understanding. Clarendon Press, Oxford.

Martini, C. (2014). Experts in science: a view from the trenches. Synthese, 191:3-15.

Matheson, J. (2015). The Epistemic Significance of Disagreement. Palgrave Macmillan, Basingstoke.

Mayo-Wilson, C., Zollman, K. J., and Danks, D. (2011). The Independence Thesis: When Individual and Social Epistemology Diverge. Philosophy of Science, 78:653-677.

McGrew, L. (2016). Evidential diversity and the negation of h: A probabilitstic account of the value of varied evidence. Ergo, 3(10).

Myrvold, W. C. (1996). Bayesianism and Diverse Evidence: A Reply to Andrew Wayne. Philosophy of Science, 63:661-665.

Neta, R. (2002). S Knows That P. Nous, 36:663-681.

Olsson, E. J. (2005). Against Coherence: Truth, Probability, and Justification. Oxford University Press, Oxford.

Schupbach, J. N. (2008). On the alleged impossibility of bayesian coherentism. Philosophical Studies, 141:323-331.

Schupbach, J. N. (2011). New hope for shogenji's coherence measure. British Journal for the Philosophy of Science, 62:125-142.

Shogenji, T. (1999). Is coherence truth-conducive? Analysis, 59:338-345.

Shogenji, T. (2001). Reply to akiba on the probabilistic measure of coherence. Analysis, 61:147-150.

Strevens, M. (2003). The Role of the Priority Rule in Science. Journal of Philosophy, 100:55-79.

Thagard, P. (1997). Collaborative Knowledge. Nous, 31:242-261.

Wayne, A. (1995). Bayesianism and Diverse Evidence. Philosophy of Science, 
62:111-121.

Wheeler, G. (2009). Focused correlation and confirmation. British Journal for the Philosophy of Science, 60:79-100.

Whewell, W. (1858). Novum Organum Renovatum. John W. Parker and Son, London, 3rd edition.

Williams, B. (1973). Deciding to believe. In Problems of the Self, pages 136151. Cambridge University Press, Cambridge.

Wray, K. B. (2002). The Epistemic Significance of Collaborative Research. Philosophy of Science, 69:150-168.

Zagzebski, L. (2007). Ethical and Epistemic Egoism and the Ideal of Autonomy. Episteme, 4:252-263.

Zagzebski, L. (2013). Intellectual autonomy. Philosophical Issues, 23:244-261.

Zollman, K. (2007). The Communication Structure of Epistemic Communities. Philosophy of Science, 74:574-587.

Zollman, K. J. (2010). The Epistemic Benefit of Transient Diversity. Erkenntnis, 72:17-35. 